\title{
Anomalous plantar intrinsic foot muscle attaching to the medial longitudinal arch: possible mechanism for medial nerve entrapment: a case report
}

\author{
R. Claire Aland ${ }^{1,2}$ and Alana C. Sharp ${ }^{3,4^{*}}$ (1)
}

\begin{abstract}
Background: Muscular variations are potentially symptomatic and may complicate imaging interpretation. Intrinsic foot musculature and extrinsic tendon insertion variations are common. Distinct supernumerary muscles are rare. We report a novel anomalous intrinsic foot muscle on the medial longitudinal arch.

Case presentation: An accessory muscle was encountered on the medial arch of the right foot of a 78-year-old white male cadaver, between layers two and three of the foot intrinsics. It did not appear to be a slip or variant of a known foot muscle. This muscle consisted of two slips that ran transversely on the plantar aspect of the medial arch, crossing the medial transverse tarsal joint and attaching to the tuberosity of the navicular, the short and long plantar ligaments, and spring ligament.
\end{abstract}

Conclusions: The medial plantar vessels and nerve passed from deep to superficial between the two slips, and this suggests a possible location for medial nerve entrapment.

Keywords: Anatomical variation, Medial longitudinal arch, Medial nerve entrapment, Plantar intrinsic muscle, Case report

\section{Background}

The plantar foot contains four layers of intrinsic muscles, interspersed with tendons of extrinsic leg muscles that insert into the foot. The first, most superficial layer contains the flexor digitorum brevis, abductor hallucis, and abductor digiti minimi; the second layer contains the quadratus plantae and four lumbricals; the third contains the adductor hallucis, flexor hallucis brevis, and flexor digiti minimi; the fourth and deepest layer contains the dorsal and plantar interossei.

*Correspondence: alana.sharp@liverpool.ac.uk

${ }^{3}$ Institute of Life Course and Medical Sciences, University of Liverpool, Liverpool, UK

Full list of author information is available at the end of the article
Several muscles move the hallux, or great toe: in the first layer, the abductor hallucis $(\mathrm{AbH})$ arises from the medial calcaneus, plantar aponeurosis, and flexor retinaculum to insert distally on the medial proximal hallucial phalanx base. In the second layer, the flexor hallucis longus tendon passes deep to the flexor digitorum longus tendon to insert on the base of the distal hallucial phalanx. The third layer contains the two heads of both the adductor hallucis (AdH) and flexor hallucis brevis (FHB). The oblique head of the AdH originates laterally from the cuboid, lateral cuneiform and from the bases of the second and third metatarsals and inserts on the lateral base of the proximal hallucial phalanx, while the transverse head arises from metatarsophalangeal joints of the third-fifth digits. The medial and lateral heads of the FHB originate from the cuneiforms and plantar original author(s) and the source, provide a link to the Creative Commons licence, and indicate if changes were made. The images or other third party material in this article are included in the article's Creative Commons licence, unless indicated otherwise in a credit line to the material. If material is not included in the article's Creative Commons licence and your intended use is not permitted by statutory regulation or exceeds the permitted use, you will need to obtain permission directly from the copyright holder. To view a copy of this licence, visit http://creativecommons.org/licenses/by/4.0/. The Creative Commons Public Domain Dedication waiver (http://creativeco mmons.org/publicdomain/zero/1.0/) applies to the data made available in this article, unless otherwise stated in a credit line to the data. 
calcaneonavicular (spring) and long plantar ligaments and insert, via the medial and lateral sesamoids of the first metatarsophalangeal joint, to the proximal hallucial phalanx. In the fourth layer, the tibialis posterior tendon inserts to the navicular and medial cuneiform, and the tibialis anterior tendon inserts to the medial cuneiform and first metatarsal. The medial plantar nerve supplies the $\mathrm{AbH}$ and $\mathrm{FHB}$, while the deep branch of the lateral plantar nerve supplies the AdH [16].

Muscular variations are potentially symptomatic and may complicate imaging interpretation. Intrinsic foot musculature and extrinsic tendon insertion variations are common [12-14, 17]. Distinct supernumerary muscles are rare. We report a novel anomalous intrinsic foot muscle on the medial longitudinal arch, which may be a variant of $\mathrm{AdH}$.

\section{Case presentation}

This anomalous muscle (Fig. 1) was discovered by dissection at the School of Rural Medicine, University of New England, Australia. The dissection was approved by the UNE Human Ethics Committee (approval number:
HE15-212). This muscle was present in the right medial arch of a 78-year-old white male cadaver. There was no equivalent muscle in the left foot. The foot did not have scars or pathology beyond age-related degeneration. No other variations were noted in the intrinsic or extrinsic musculature of either foot.

The anomalous muscle was formed from two slips (proximal and distal) on the plantar medial longitudinal arch, located deep to the $\mathrm{AbH}$, and the tendons of the flexor digitorum longus and flexor hallucis longus. The tibialis anterior tendon inserted distal to the distal slip. Both slips passed superficially to the tibialis posterior tendon insertion and were proximal to the FHB origin.

The distal slip traveled transversely, while the proximal slip ran obliquely from the lateral attachment proximally to the medial attachment distally. The distal slip had muscular attachments to the short plantar ligament and some aponeurotic fibers to the long plantar ligament. The proximal slip had muscular attachments to the short plantar and spring ligaments, attaching near, yet remaining separate from, the tibialis posterior tendon, proximal tendinous origin of the lateral head of the FHB, and
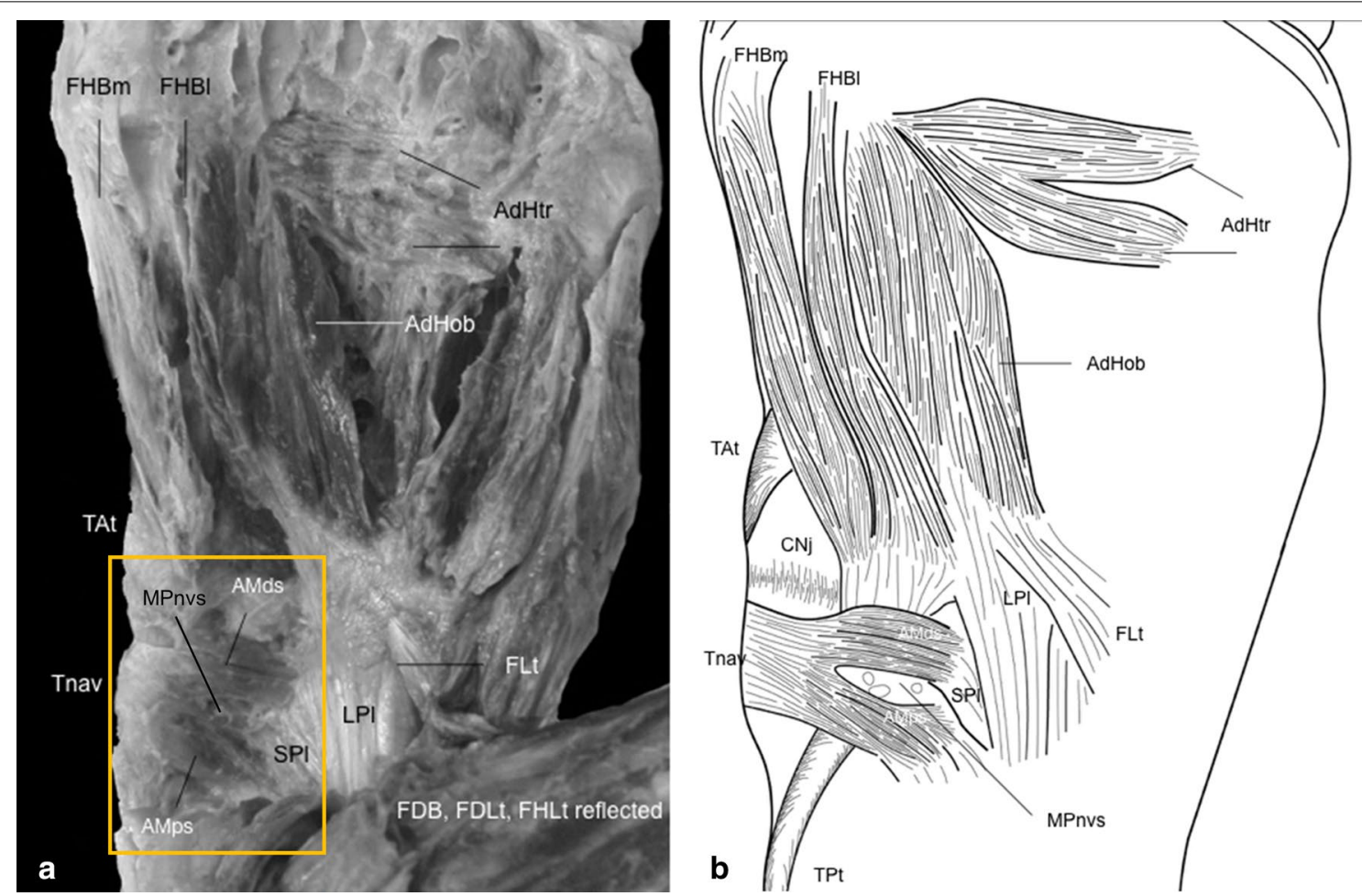

Fig. 1 Anomalous muscle slips attach to the medial longitudinal arch: a photograph; b schematic drawing. Abductor hallucis (AbH) is removed, and the flexor digitorum longus (FDL) and flexor hallucis longus (FHL) tendons and the flexor digitorum brevis (FDB) have been reflected. AdHob Adductor hallucis oblique head; AdHtr adductor hallucis transverse head; AMds anomalous muscle distal slip; Amps anomalous muscle proximal slip; CNj cuneonavicular joint; FDB flexor digitorum brevis FDLt flexor digitorum longus tendon; FHLt reflected flexor hallucis longus tendon, reflected; FLt fibularis longus tendon; LPI long plantar ligament; MPnvs medial plantar nerves and vessels, SPI short plantar ligament, Tat tibialis anterior tendon, Thav navicular tuberosity, TPt tibialis posterior tendon 
oblique head of the AdH. Proximal and distal slips combined to attach to the navicular tuberosity as aponeurotic fibers. Some extended to the tibionavicular portion of the deltoid ligament.

The medial plantar vessels and nerve passed from deep to superficial between the two slips (Fig. 1b). Small branches from these supplied the anomalous muscular slips.

\section{Discussion and conclusions}

We present an anomalous muscle of the medial longitudinal arch that to our knowledge has not been previously reported. The position suggests a possible variation of an intrinsic muscle associated with the great toe or medial foot. Numerous variations in the abductor hallucis $(\mathrm{AbH})[1,4]$ and adductor hallucis $(\mathrm{AdH})[2,8]$ have been reported. For example, variant muscular slips of the $\mathrm{AbH}$ attaching to the proximal phalanges of the second and third toes [12], flexor hallucis longus tendon, or skin of the hallux [15] have been reported. Distally, AbH may also attach to the underlying tarsals, metatarsals, and fascial septae [6]. However, these are not discrete slips and are oriented with the main muscle belly rather than perpendicular to it as with the anomalous muscles described here.

Variable insertions of either head of the AdH into the first proximal phalanx have been described as opponens hallucis $(\mathrm{OH})$ [12]. Macalister [15] regarded $\mathrm{OH}$ as frequently present in humans, either as a free slip or, more commonly, fused with the AdH. However, modern anatomical texts do not regard $\mathrm{OH}$ as a distinct muscle [16], nor do those variants resemble the $\mathrm{OH}$ of non-human primates $[3,5,9]$. However, all these muscle variants are described as running longitudinally, arising from the medial cuniform or navicular and inserting to the first metatarsal shaft or proximal hallucial phalanx base rather than running transversely.

The interpretation of the present anomalous muscle as an $\mathrm{OH}$ is also not clearly supported when considering development. The medial foot muscles develop from the superficial and deep layers of the plantar muscular blastema [7]. The superficial layer differentiates first, forming the $\mathrm{AbH}$. The deep layer of the blastema splits to form two adjacent components-the medial and lateral heads of the FHB (initially formed as independent muscles) and a component that becomes the variable first plantar interosseous muscle [7]. In comparison, the primordia for three consistent hand muscles are formed-the deep and superficial heads of the flexor pollicis brevis and the opponens pollicis. The latter lies lateral to the flexor pollicis brevis and deep to the abductor pollicis brevis. An equivalent $\mathrm{OH}$ does not occur in humans; the FHB and $\mathrm{AdH}$ variants proposed as such are more lateral and distal. The variable component of the deep plantar blastema may become continuous with the $\mathrm{AdH}$ oblique head or the first dorsal interosseous, or be considered the deep head of the FHB [7]. Čihák [7] termed this the musculis interosseous plantaris hallucis rather than accepting equivalence to the $\mathrm{OH}$.

Direct comparison of development, anatomical variants, or function with non-human primates is difficult, as human bipedalism and loss of the hallucial grasp have resulted in musculoskeletal alterations in the foot [11]. However, this variant does not appear situated to produce opposition of the hallux and may act as a tensor of the medial longitudinal planar arch. The transverse orientation of the distal slip, with its attachments on the short and long plantar ligaments and navicular tuberosity, combined with the oblique orientation of the proximal slip, attaching to the proximal tendinous origin of the lateral head of the FHB, oblique head of $\mathrm{AdH}$, and navicular tuberosity, suggests a relationship with the medial transverse tarsal joint and plantar calcaneonavicular (spring) ligament, potentially providing active support to stiffen the arch during locomotion. However, its small size belies meaningful movement at this area.

The medial plantar vessels and nerve pass from deep to superficial between the two slips (Fig. 1b), while giving off small branches to supply the muscular slips of this anomalous muscle. The medial plantar nerve arises under the flexor retinaculum, runs deep to the $\mathrm{AbH}$, and, with the medial plantar vessels, travels between the $\mathrm{AbH}$ and flexor digitorum brevis [16]. Medial plantar nerve entrapment between the $\mathrm{AbH}$ and navicular, in the apex of the medial longitudinal arch, may result in medial plantar neuropraxia (Jogger's foot) [10]. Passage of the medial plantar vessels and nerve between the two muscular slips here suggests a possible mechanism for nerve entrapment as these muscular slips contract.

Knowledge of variant foot muscles is important because of potential for pathology and for imaging interpretation. While analogous muscle slips described in non-human primates and the developmental origin of this anomalous muscle remain unclear, the location suggests a possible mechanism for medial nerve entrapment and function in supporting the medial longitudinal arch.

\section{Acknowledgements \\ The authors thank Profs. A. Murphy and G. Ranmuthugala for approving and supporting this work and two reviewers for their helpful comments on this manuscript. We also sincerely thank those who donated their bodies to sci- ence, and their families, so that this anatomical research could be performed.}

\section{Authors' contributions}

All authors designed the study, conducted the research, and wrote and approved the final manuscript.

Funding

No funding to declare 


\section{Availability of data and materials}

Data sharing is not applicable to this article as no datasets were generated or analyzed during the current study.

\section{Ethics approval and consent to participate}

The research detailed in this manuscript was subject to ethical review and approval by the Human Research Ethics committee at the University of New England at which it was performed and adhered to the legal requirements of Australia (approval number HE15-212).

\section{Consent for publication}

Written informed consent was obtained from the patient for publication of this case report and any accompanying images. A copy of the written consent form is available for review by the Editor-in-Chief of this journal.

\section{Competing interests}

The authors declare that they have no competing interests.

\section{Author details}

1 School of Rural Medicine, University of New England, Armidale, NSW, Australia. ${ }^{2}$ School of Biomedical Sciences, University of Queensland, Brisbane, QLD, Australia. ${ }^{3}$ Institute of Life Course and Medical Sciences, University of Liverpool, Liverpool, UK. ${ }^{4}$ School of Science \& Technology, University of New England, Armidale, NSW, Australia.

Received: 14 April 2020 Accepted: 11 January 2021

Published online: 13 February 2021

\section{References}

1. Agawany AE, Meguid EA. Mode of insertion of the abductor hallucis muscle in human feet and its arterial supply. Folia Morphol. 2010;69:54-61.

2. Arakawa T, Tokita K, Miki A, Terashima T. Anatomical study of human adductor hallucis muscle with respect to its origin and insertion. Ann Anat. 2003;185(6):585-92.

3. Boyer EL. The musculature of the inferior extremity of the orang-utan, Simia satyrus. Am J Anat. 1935;56:192-256.

4. Brenner E. Insertion of the abductor hallucis muscle in feet with and without hallux valgus. Anat Rec. 1999;254:429-34.
5. Brooks HSJ. On the short muscles of the pollex and hallux of the anthropoid apes, with special reference to the opponens hallucis. J Anat Physiol. 1887:22:78-95

6. Chittoria RK, Pratap H, Yekappa SH. Abductor hallucis: Anatomical variation and its clinical implications in the reconstruction of chronic nonhealing ulcers and defects of foot. Adv Wound Care. 2015;4(12):719-23.

7. Čihák R. Ontogenesis of the Skeleton and Intrinsic Muscles of the Human Hand and Foot. In: Advances in anatomy, embryology, and cell biology. Berlin Heidelberg GmbH: Springer-Verlag; 1972. p. 5-194.

8. Cralley JC, Schuberth JM. The transverse head of adductor hallucis. Anat Anz. 1979;146:400-9.

9. Diogo R, Shearer B, Potau JM, Pastor JF, De Paz FJ, Arias-Martorell J, Turcotte C, Hammond A, Vereecke E, Vanhoof M, Nauwelaerts S. Photographic and Descriptive Musculoskeletal Atlas of Bonobos. New York: Springer; 2017

10. Ferkel E, Davis WH, Ellington JK. Entrapment neuropathies of the foot and ankle. Clin Sports Med. 2015;34(4):791-801.

11. Harcourt-Smith WE, Aiello LC. Fossils, feet and the evolution of human bipedal locomotion. J Anat. 2004;204(5):403-16.

12. Kafka RM, Aveytua IL, Fiacco RC, Ream GM, DiLandro AC, D'Antoni A. Intrinsic Muscles of the Foot. In: Tubbs RS, Shoja MM, Loukas M, editors. Bergman's Comprehensive Encyclopedia of Human Anatomic Variation. New York: Wiley; 2016. p. 438-48.

13. Kura H, Luo Z-P, Kitaoka HB, An K-N. Quantitative analysis of the intrinsic muscles of the foot. The Anatomical Record. 1997;249(1):143-51.

14. Lambert HW. Leg muscles. In: Tubbs RS, Shoja MM, Loukas M, editors. Bergman's comprehensive encyclopedia of human anatomic variation. John Wiley \& Sons; 2016. p. 421-47.

15. Macalister A. Macalister on muscular anomalies in human anatomy. Trans R Ir Acad. 1875;25:34.

16. Standring S (ed.). Gray's Anatomy 40th edition. Elsevier Churchill Livingstone; 2008. p. 1429-1462.

17. Wong YS. Influence of the abductor hallucis muscle on the medial arch of the foot: a kinematic and anatomical cadaver study. Foot Ankle Int. 2007:28(5):617-20.

\section{Publisher's Note}

Springer Nature remains neutral with regard to jurisdictional claims in published maps and institutional affiliations.
Ready to submit your research? Choose BMC and benefit from:

- fast, convenient online submission

- thorough peer review by experienced researchers in your field

- rapid publication on acceptance

- support for research data, including large and complex data types

- gold Open Access which fosters wider collaboration and increased citations

- maximum visibility for your research: over $100 \mathrm{M}$ website views per year

At $\mathrm{BMC}$, research is always in progress.

Learn more biomedcentral.com/submissions 